\title{
Fossile Bodenbildungen auf quartären Flußschottern an der Mittellahn und ihre Bedeutung für die Terrassenstratigraphie
}

\author{
Von Klaus Heine, Bonn
}

Mit 2 Abbildungen

$\mathrm{Z} u$ s a mmenfassung. Umfangreiche Untersuchungen der fossilen Böden auf Lahnterrassenschottern im Gelände und im Labor haben gezeigt, daß unterschiedliche Bodenbildungen für die einzelnen Terrassengruppen charakteristisch sind. Sie können bei der Terrassenstratigraphie von Nutzen sein.

$\mathrm{Summary}$. The weathering of the gravels of the terraces of the river Lahn is described. Comprehensive investigations have shown that different types of fossil soils are characteristic for the different groups of terraces. The fossil soils can be used with regard to a stratigraphy of the terraces.

Die Hänge des Mittellahntals werden im Bereich Marburgs von verschiedenen Terrassenniveaus gegliedert. Sieben morphologisch einwandfrei auszugliedernde Terrassenkomplexe konnten ermittelt werden. Ein schematisches Querprofil zeigt die Anordnung der Terrassen in ihrer vertikalen Abfalge (Abb. 1). Nur die Terrassengruppen von der Talfüllung bis zur 40-60-m-Terrasse besitzen mehr oder weniger mächtige fluviatile Schotterablagerungen. Die 70-80-m-Terrasse ist lediglich als Felsterrasse ausgebildet. Im flächenhaft ausgebildeten 300-m-Niveau sind vereinzelt Leseschotter anzutreffen.

Bisher wurden keine Versuche unternommen, die Terrassenreste zeitlich zu fixieren. Weder das Alter der Terrassen noch die Ursachen ihrer Bildung waren bekannt. Petrographische und Schwermineral-Analysen der Flußschotter — von LANG 1955 ausgeführt konnten die stratigraphische Stellung der Terrassensedimente nicht klären. In der Regel sind die Schotterablagerungen der Terrassen äußerst geringmächtig - die Beträge schwanken zwischen wenigen Dezimetern bis maximal 6,5 $\mathrm{m}$ in der Hauptterrasse - . Aus diesem Grunde sind ältere Terrassensedimente oft so stark verwittert, daß weder die petrographische noch die Schwermineral-Analyse Aussagen liefern kann, abgesehen von der allgemeinen Tendenz, daß sich der Verwitterung gegenüber resistente Komponenten relativ anreichern, und zwar in den höher gelegenen Terrassen am stärksten.

Auch eine genaue Untersuchung und Betrachtung der Deckschichten über den verschiedenen Terrassensedimenten führt nicht wesentlich weiter. Wohl ist zu beobachten, daß die Schotter der Talaue von einem Auelehm (Ausediment) und die niedrigste $(2-4 \mathrm{~m})$, mor-

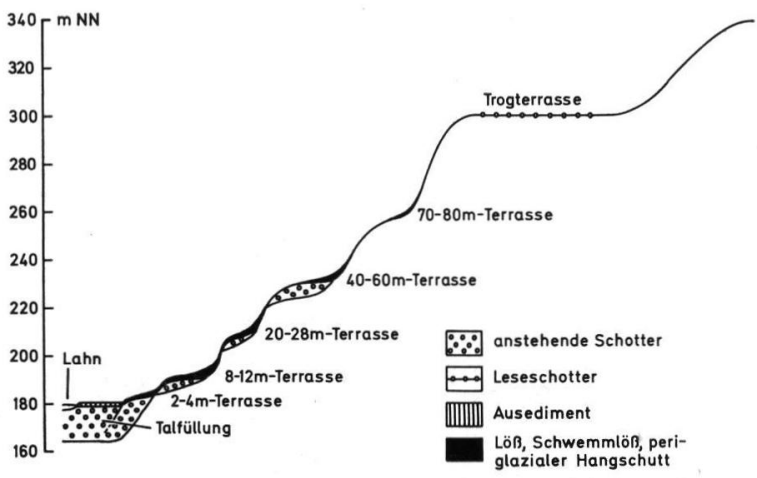

Abb. 1. Schematisches Talquerprofil der Lahn bei Marburg. 
phologisch ausgewiesene Terrasse in der Regel von umgelagertem Löß bedeckt sind; die anderen Terrassenkörper zeigen im allgemeinen die gleichen Verhältnisse hinsichtlich der Deckschichten, nämlich eine Überdeckung mit Solifluktionsschutt und/oder Schwemmlöß oder Löß. Da die Deckschichten aufgrund der topographischen Verhältnisse kein älteres als würmglaziales Alter aufweisen, läßt sich mit Hilfe der Deckschichten nur ein Mindestalter ermitteln. Präwürmzeitliche Deckschichten über Terrassenschottern sind wegen der geringen Ausmaße der Terrassenreste und wegen der oft zum Lahntal hin einfallenden Terrassenoberflächen bereits vor der Ausbildung der jüngeren Deckschichten abgetragen worden. In keinem Fall wurde im Mittellahntal über fluviatilen Schottern eine Abfolge von Deckschichten bekannt, die a priori auf eine mindel- oder gar günzzeitliche Sedimentation der liegenden Schotter hindeutete.

War es somit also nicht möglich, eine genaue Datierung der Terrassensedimente anhand der morphologischen Befunde, der Deckschichten, der petrographischen sowie Schwermineral-Analyse vorzunehmen, so blieb die Hoffnung bei einer genauen Untersuchung der Bodenbildungen auf den Schottern selbst Anhaltspunkte für eine stratigraphische Einordnung der Terrassen zu gewinnen. Dabei wurden die Beobachtungen im Gelände durch Untersuchungen im Labor ergänzt. Bei der Probenentnahme wurde darauf geachtet, daß das Material einem Schotterkörper entnommen wurde, der aufgrund seiner petrographischen Verhältnisse als typisch für den Mittellahnlauf anzusehen ist; dabei spielen Gerölle aus dem Rheinischen Schiefergebirge und den Buntsandsteingebieten um Marburg eine besondere Rolle, während Komponenten aus Gebieten des sedimentären und basaltischen Tertiärs nur selten beigemischt sind. Bestimmt wurden die Korngrößen, pH- und Farbwerte, $\mathrm{CaCO}_{3}$-Gehalt sowie Humusanteil, hydrolytische und Austausch-Azidität, S-, T-, T-S- und V-Wert, die Tonmineralgarnitur mit Hilfe der röntgenographischen Analyse und des Elektronenmikroskops. Die Ergebnisse erlauben eine Datierung der Sedimente an der Mittellahn und lassen eine Parallelisierung der Lahnterrassen bei Marburg mit den Mittelrheinterrassen zu.

Die Beobachtungen im Gelände sowie die Untersuchungen im Laboratorium bestätigen die Annahme einer glazial-klimatischen Terrassenaufschotterung (HEINE 1970). Als jüngste Bildungen dürfen die Schotter der Talfüllung angesehen werden (vgl. hierfür und für die folgenden Ausführungen Abb. 2). Sie werden bei Marburg von ca. 2 bis maximal $4 \mathrm{~m}$ mächtigen Auelehmen bedeckt. Die Ablagerung der Auelehme ist durch pollenanalytische Untersuchungen durch LANG 1954-56 und neuerdings durch MäcKeL (1969), dazu durch die Verknüpfung mit Laacher See-Tuffen seit dem Alleröd nachgewiesen. Verschiedene Phasen der Akkumulation, der Erosion und der Bodenbildung sind im Auelehm zu beobachten; diese Vorgänge sind allerödzeitlich und jünger. Die von den Ausedimenten bedeckten Schotter sind demnach älter. Die Schotter selbst sind unverwittert; ihre Farbe ist grau, graubraun oder rötlichbraun, je nachdem, inwieweit infolge des Grundwassereinflusses Eisenoxide und -hydroxide ausgeschieden wurden. Der Tongehalt der Schotter der Talfüllung beträgt immer unter $1 \%$; in der Tonsubstanz herrschen Hydroglimmer, Quarz und Illit vor, während Kaolinit, Montmorillonit und Vermiculit als Nebenbestandteile auftreten. Die Schotter sind kalk- und humusfrei; sie haben eine schwach saure Reaktion, eine mäßige hydrolytische Azidität und Austauschazidität.

Ebenfalls unverwittert sind die Schotter der 2-4-m-Terrasse, die jedoch häufig von umgelagertem Löß bedeckt werden. Die holozäne Verwitterung führte auf den Deckschichten zu einer Braunerdebildung, deren $\mathrm{B}_{\mathrm{v}}$-Horizont gelegentlich - bei geringmächtiger Schwemmlößauflage - bis in die Kiese der 2-4-m-Terrasse eindringen kann. Die Schotter der Talfüllung sowie der 2-4-m-Terrasse sind würmzeitliche Bildungen und infolge der Überlagerungen durch Ausedimente einerseits und Schwemmlöß andererseits post-würmzeitlich nicht verwittert. Die Schotter entsprechen damit der oberen (=älteren) 

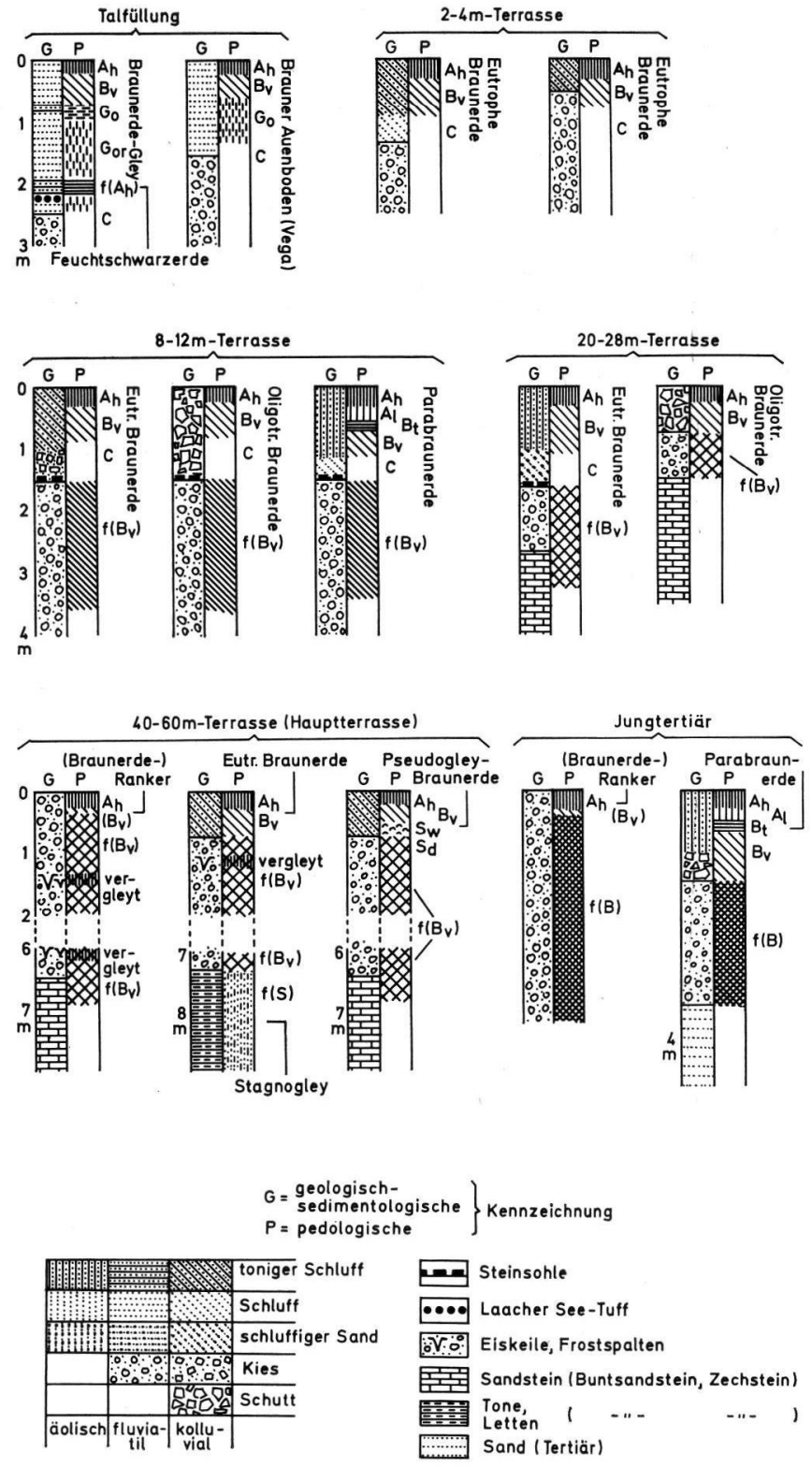

Fossile Bodenbildungen auf Terrassenschottern:
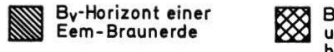

Bv-Horizont holsteinund cromerzeitlicher braunlehmartiger gelblichroter Interglazialböden

Abb. 2. Die häufigsten Bodenbildungen auf Terrassenschottern und Deckschichten (schematisch). 
Niederterrasse des Mittelrheintales. Die untere (=jüngere) Niederterrasse ist im Marburger Raum nicht ausgebildet; analoge Bildungen dazu sind Teile der Ausedimente.

Merkliche Unterschiede hinsichtlich des Verwitterungsgrades gegenüber den Schottern der Talfüllung und der 2-4-m-Terrasse zeigen die kiesigen Ablagerungen in ca. 8-12 m über der heutigen Talaue. Die Sedimente dieser Terrassengruppe werden überall von Solifluktionsschutt und/oder Schwemmlöß oder Löß überdeckt. Die Bodenbildungen der Deckschichten sind post-pleistozän; es handelt sich um vorwiegend oligotrophe Braunerden auf dem Solifluktionsmaterial des Buntsandsteins, um eutrophe Braunerden auf Schwemmlöß, der oft einen gewissen Anteil an Verwitterungsmaterial des Buntsandsteins enthält, und um eutrophe Braunerden bis Parabraunerden auf Lößablagerungen. Die Schotter der 8-12-m-Terrasse, die einen relativ hohen Anteil an Buntsandstein- und Zechsteingeröllen haben, zeigen in den hangenden Partien eine ziemlich gleichmäßig braune Färbung infolge einer Verwitterung, die mehrere Meter in den Schotterkörper eindringen kann. Aufgrund der pedologischen Beobachtungen im Gelände und der Laborbefunde darf die Bodenbildung typologisch als Braunerde angesprochen werden, die ursprünglich eine schwach saure Reaktion aufwies, jedoch infolge sekundärer Einwaschung von $\mathrm{Ca}$-Ionen aus den hangenden Deckschichten heute oft eine neutrale Reaktion zeigt. Die obersten Teile dieser fossilen Braunerden sind wahrscheinlich während des Würms gekappt worden, so daß nur noch Teile der $\mathrm{B}_{\mathrm{V}}$-Horizonte erhalten sind, die unvermittelt gegen die Deckschichten grenzen. Der Tongehalt am Gesamtsediment schwankt zwischen 1,5 und 8\%, was als Folge der unterschiedlich starken Abtragung der obersten Bodenhorizonte anzusehen ist. Unter den Tonmineralen tritt der Kaolinit zugunsten des Hydroglimmers etwas stärker hervor. Die ursprüngliche Entwicklungstiefe der fossilen Braunerden der 8-12-m-Terrassen muß zwei Meter überschritten haben; die Bören können vereinzelt auch schwach ausgebildete, braunlehmartige Merkmale zeigen, besonders wenn Silikatgesteine gegenüber Sandsteinen im Ausgangsmaterial stark überwiegen; all diese Befunde deuten auf eine Bodenbildung nicht mehr während des Würmglazials und Holozäns hin, sondern während des EemInterglazials. Vergleiche mit eemzeitlichen Bodenbildungen auf mittelrheinischen Terrassenschottern bestätigen diese Annahme. Die 8-12-m-Terrassen der Lahn sind demnach rißzeitliche Bildungen und entsprechen der unteren Mittelterrasse des Mittelrheins.

Im Niveau der 20-28-m-Terrassen sind Schotterablagerungen recht selten. Nur vereinzelt wurden geringmächtige fluviatile Sedimente unter Deckschichten aus Solifluktionsmaterial und/oder Schwemmlöß oder Löß angetroffen. An keinem Ort betrug die Schottermächtigkeit dieser Terrassengruppe über einen Meter; zudem gab es überall Anzeichen für eine postsedimentäre Umlagerung des Materials. Teilweise befanden sich Schotter dieser Terrassen im Niveau der rißzeitlichen 8-12-m-Terrassen. Aufgrund der Deckschichten, die sich nicht von denjenigen der 8-12-m-Terrassen unterscheiden, kommt für die Ablagerung der Schotter der 20-28-m-Terrasse ein mindestens rißzeitliches Alter in Frage. Die kräftige Verwitterung der Sedimente jedoch hebt sich deutlich von den Bodenbildungen der tieferen Terrassenschotter ab. Der Verlehmungsgrad - der Tongehalt am Gesamtsediment schwankt im Durchschnitt zwischen 10 und 20\% -, die große Plastizität, die recht intensive gelblich-rote Farbe (5 YR 4/8), die saure bis schwach saure Bodenreaktion, die mäßigen Austauscheigenschaften, die hochplastische, außerordentlich leicht verschlämmende, durch kolloidale Kieselsäure peptisierte Tonsubstanz und die Tonmineralgarnitur, in der eine deutliche Zunahme des Kaolinits und eine Abnahme der Hydroglimmer sowie der Illite zu verzeichnen ist, dazu eine wesentlich bessere Kristallisation der Kaolinitminerale, lassen einen Boden erkennen, der den von PAAs (1961) für die rheinische obere Mittelterrasse beschriebenen Bodentypen sehr ähnlich ist und daher ebenfalls bodentypologisch als „braunlehmartiger gelblich-roter Interglazialboden“ bezeichnet werden soll. Er gehört zur Bodenklasse der von Kubiena (1953) beschriebenen „bolusartigen Silikatböden". Braunlehme haben eine große Stabilität, weshalb sich ihre Eigenart in den 
meisten Ablagerungen und Reliktvorkommen weitgehend erhalten hat. Nur so ist es zu erklären, daß die ursprüngliche braunlehmartige Verwitterung der 20-28-m-Terrassenschotter selbst in geringmächtigen Ablagerungen und nach geringfügigen, zumeist solifluidalen Umlagerungen noch zu erkennen ist. Diese Braunlehme sind das Ergebnis einer intensiven Bodenbildung während relativ langdauernder, warmer und feuchter Interglazialzeiten. Für ihre Bildung muß aufgrund der pedologischen Befunde das Holstein-Interglazial angenommen werden. Die Sedimente der 20-28-m-Terrassen haben somit mindelzeitliches Alter und können mit der mittelrheinischen oberen Mittelterrasse parallelisiert werden.

Die Schotter der 40-60-m-Terrassen (Hauptterrassen) werden durch einen ähnlichen, jedoch wesentlich stärker ausgeprägten „braunlehmartigen, gelblich-roten Interglazialboden“ gekennzeichnet. In dem intensiv verwitterten, plastischen Bodenmaterial beträgt der Tonanteil über 20\%, z. T. über 30\%. Auch dieser Boden ist kalk- und humusfrei; die Bodenreaktion ist sauer; die Austauscheigenschaften sind schlecht. Das Kaolinit/Illit-Verhältnis ist weiter zugunsten des Kaolinits verschoben. Die Entwicklungstiefe dieser braunlehmartigen Bodenbildungen durchdringt den gesamten Hauptterrassenschotterkörper, dessen Mächtigkeit sechs Meter und mehr betragen kann. Nur die Summation mehrerer, relativ warmer und feuchter Interglazialzeiten (Cromer und Holstein) vermag zu einer derart intensiven Ausbildung eines braunlehmartigen Bodens auf Terrassenschottern führen. Ein gleiches Alter der Bodenbildungen auf den 20-28-m- und den Hauptterrassensedimenten ist aufgrund der pedologischen, aber auch der morphologischen Kriterien ausgeschlossen. Dieser gelblich-rote braunlehmartige Interglazialboden der Hauptterrassensedimente entspricht der Verwitterung der jüngeren Hauptterrasse des Mittelrheingebietes. Ein gleiches, wahrscheinlich günzzeitliches Alter der Ablagerung der Schotter der rheinischen jüngeren Hauptterrasse einerseits und der 40-60-m-Terrasse der Mittellahn andererseits scheint daher sehr naheliegend. Das aus dem Marburger Raum bis zum Mittelrheintal zu verfolgende Hauptterrassenniveau bestätigt die bodenkundlichen Untersuchungen und Folgerungen. - Die Deckschichten der Lahn-Hauptterrasse zeigen oft die Spuren einer Pseudovergleyung als Folge der wasserstauenden, stark verwitterten Schotterunterlage. Auch in den Schottern selbst sind Anzeichen einer Pseudovergleyung - besonders im Bereich ehemaliger Eisspaltennetze - häufig zu beobachten. Werden die Hauptterrassenschotter nicht von Deckschichten überlagert, so ist als holozäne Bodenbildung oft ein Braunerderanker ausgebildet.

Im Niveau der vermutlich prägünzzeitlichen, altquartären 70-80-m-Terrassen sind weder Schotter noch fossile Bodenbildungen vorhanden.

Trogterrassen befinden sich ca. $100 \mathrm{~m}$ über dem rezenten Talboden. Auch hier fehlen fluviatile Ablagerungen sowie fossile Bodenbildungen. Anhand morphologischer Untersuchungen konnten diese Terrassenreste jedoch in das Pliozän datiert werden.

Der Vollständigkeit halber sollen auch noch die Bodenbildungen behandelt werden, die nicht mehr dem Quartär zuzurechnen sind. Auf den tertiären Verebnungsflächen, vorwiegend im Bereich der heutigen Wasserscheiden (zwischen Kellerwald und Vogelsberg), befinden sich zuweilen Bodensedimente, die durch ihre intensiv rote Farbe (2.5 YR 4/6) auffallen. Sie sind kalk- und humusfrei und haben eine saure Reaktion, jedoch bei Lößüberlagerung infolge der Einwaschung von $\mathrm{Ca}$-Ionen oft eine schwach saure bis neutrale Reaktion. Ihre Austauscheigenschaften sind schlecht, können aber — wenn im Pleistozän kalkhaltige Deckschichten vorhanden waren - durchaus mäßig werden. Der Tonanteil am Gesamtsediment beträgt in der Regel um 30\%, dem Boden fehlt aber die auffallende Schwere, Klebrigkeit und Verschlämmbarkeit der pleistozänen braunlehmartigen Interglazialböden. Auch die Plastizität ist wesentlich geringer. Das Material läßt sich in der Hand leicht krümeln. In der Tonsubstanz ist fast nur noch Kaolinit vorhanden, der zudem recht gut kristallisiert ist. Der Eisenhydroxidgehalt im Schlämmstoffkomplex scheint 
gegenüber den braunlehmartigen Interglazialböden höher zu sein. Die sedimentpetrographischen sowie chemischen und tonmineralogischen Untersuchungen lassen auf einen ursprünglich latosolartigen Boden - eine Roterde - schließen, der allerdings später geringfügig überprägt und umgelagert wurde. Die Ablagerung der Flußgerölle, die im Zusammenhang mit diesen Bodenbildungen gefunden wurden, werden jungtertiären Flußläufen zugeschrieben (HEINE 1970).

Frau Marchal, die die Analysen im Laboratorium des Geographischen Institutes der Universität Bonn durchführte, und Herrn Professor Dr. Dr. E. Mückenhausen, der mir freundlicherweise die Geräte seines Institutes zur Verfügung stellte, sowie Frl. Bödewadt, die elektronenmikroskopische Aufnahmen besorgte, möchte ich auch an dieser Stelle für ihre Hilfe danken.

\section{Schriftum}

Heine K.: Fluß- und Talgeschichte im Raum Marburg. - Bonner Geogr. Abh., 42, Bonn 1970. Kubiena, W.: Bestimmungsbuch und Systematik der Böden Europas. — Stuttgart 1953.

LANG, H. D.: Ein Alleröd-Profil mit eingelagertem Laacher See-Tuff bei Marburg/Lahn. - N. Jb. Geol. Paläont., Mh. 1954 (8), 362-372, Stuttgart 1954.

- : Zur Flußgeschichte der Lahn. Ergebnis geröllanalytischer Untersuchungen in der Umgebung von Marburg/Lahn. - Diss. Marburg 1955, unveröffentlicht.

- : Jungpleistozäne Torfe im nördlichen Hessen. - Notizbl. hess. L.-Amt Bodenforsch., 84, 245-251, Wiesbaden 1956.

MäCKEL, R.: Untersuchungen zur jungquartären Flußgeschichte der Lahn in der Gießener Talweitung. - Eiszeitalter u. Gegenwart, 20, 138-174, OOhringen 1969.

PaAs, W.: Rezente und fossile Böden auf niederrheinischen Terrassen und deren Deckschichten. Eiszeitalter u. Gegenwart, 12, 165-230, Ơhringen 1961.

Manuskript eingeg. 8. 10. 1970.

Anschrift des Verf.: Dr. Klaus Heine, 53 Bonn, Geographisches Institut der Universität, Franziskanerstraße 2. 